\title{
A new species of Polyosma Blume (Escalloniaceae) and notes on a revision of the genus in Peninsular Malaysia and Singapore
}

\author{
L.G. Saw \\ Penang Botanic Gardens, \\ Jalan Kebun Bunga, \\ 10350 Penang, Malaysia \\ sawlg55@gmail.com
}

\begin{abstract}
Twelve species of Polyosma are recognised for Peninsular Malaysia and Singapore, of which only three species are in Singapore. One new species is described, the taxonomy of two species is clarified with five names synonymised, and all names are typified. Provisional conservation assessments are provided for each species.
\end{abstract}

Keywords. Conservation assessment, taxonomy, typification

\section{Introduction}

Polyosma Blume is a moderately large genus of about 80 species (Lundberg, 2016a). It is mainly Southeast Asian in distribution, occurring from northeastern India to southern China to New Caledonia and the east coast of Australia. The genus Polyosma was first published by Blume (1826) where he described three species, P. ilicifolia Blume, $P$. integrifolia Blume and $P$. serrulata Blume from Indonesia (Netherlands Indies). Wallich (in Roxburgh, 1824) had already earlier suspected a new genus when he described Itea ? fragrans Wall., which Bennett (1840) subsequently transferred to Polyosma fragrans (Wall.) Benn. The more important revisions or treatments for the genus in the Malesian region include Clarke (1879), King (1897), Ridley (1917, 1922), Stone (1980) and Saw (1989) for Malaya; Merrill (1905, 1912, 1915, 1916, 1919, 1923) and Elmer (1913, 1939) for the Philippines; Stapf (1894), Schlechter (1914), Merrill (1921, 1928), Heine (1953) and Saw (2004) for Borneo (mainly Sabah and Sarawak); Blume (1850) and Backer \& Bakhuizen van den Brink Jr (1964) for Java. Craib (1928, 1929) and Esser \& Saw (2015) described new species for the genus in Thailand and, more recently, Esser (2017) revised the genus (under Polyosmaceae) for Thailand.

Polyosma and several other genera were formerly placed in the broadly defined Saxifragaceae. Recent molecular phylogeny shows that Saxifragaceae is polyphyletic and elements of it have been moved to several families, e.g. Escalloniaceae, Grossulariaceae, Hydrangeaceae, Iteaceae and Polyosmaceae following different circumscriptions (Soltis \& Soltis, 1997; Stevens, 2001 onwards; APG IV, 2016; Lundberg, 2016a, 2016b). Following APG IV (2016), the genera found in Peninsular 
Malaysia and Singapore that were formerly placed in Saxifragaceae s.1. are now placed in Escalloniaceae (Polyosma), Iteaceae (Itea L.) and Hydrangeaceae (Hydrangea Gronov., formerly Dichroa Lour.). This treatment follows the APG IV classification by recognising Polyosma in Escalloniaceae. In Peninsular Malaysia and Singapore, the family is now represented only by Polyosma.

In preparation for a revision of the Escalloniaceae for the Flora of Peninsular Malaysia and Flora of Singapore, Polyosma has been revised for both Peninsular Malaysia and Singapore. Twelve species are recognised. Of these, eight are endemic in Peninsular Malaysia of which three are considered here to be narrowly endemic (Polyosma fasciculata Ridl., P. glaucescens Ridl. and P. nullii B.C.Stone). Only three species extend into Singapore, viz. Polyosma fragrans, P. kingiana Schltr. and $P$. ridleyi King.

Polyosma is a distinctive genus, easily recognisable by its oppositely decussate leaf arrangement, being exstipulate, and leaf margins that are sometimes toothed. It has a terminal racemose inflorescence, bisexual flowers, pedicel subtended by 3 bracteoles, central bracteole often longer and placed above the adjacent ones, petals 4, valvate, sepals 4, ovary inferior, 1-locular, style columnar, ovules numerous, placentation parietal. Fruits are single-seeded drupes, calyx often persistent at the apex; endocarp smooth and sometimes sulcate.

\section{Material and methods}

The revision is based on observations of specimens from A, B, BM, E, K, KEP, KLU, L, M, P, SAN, SAR, SING and TCD (Thiers, continuously updated). All specimens have been seen unless otherwise stated. Images of type specimens were also accessed through JSTOR Global Plants, Naturalis BioPortal and Europeana websites.

The vegetation types given in the Ecology sections below follow notes on the specimens and observations in the field and the classification of Saw (2010).

Specimen and taxonomic information management for the revision was made using the BRAHMS database software (https://herbaria.plants. ox.ac.uk/bol/brahms/software). An index of exsiccatae is provided in Appendix 1, the list was generated by BRAHMS database software. Provisional conservation assessments were made on a global level based on specimen data from herbarium collections. The assessments for all species follow IUCN Red List Categories and Criteria (IUCN Standards and Petitions Subcommittee, 2017). Calculations for extent of occurrence (EOO) and area of occupancy (AOO) were made using the GeoCat tools (Bachman et al., 2011).

\section{New species and notes on the taxonomy of Polyosma species}

1. Polyosma collina Saw, sp. nov. Similar to Polyosma fragrans (Wall.) Benn. but differs in having thickly coriaceous leaves and petiole less than $1 \mathrm{~cm}$ long, and fruit 
pedicel 2-3 mm long; $P$. fragrans has chartaceous leaves with petioles $>1 \mathrm{~cm}$, and fruit pedicel 4-10 mm long. - TYPE: Malaysia, Pahang, Cameron Highlands, Gn. Jasar, 2 March 1968, Ogata KEP 110313 (holotype KEP [181290], isotypes K, L, SING). (Fig. 1).

Polyosma fragrans auct. non (Wall.) Benn.: Ridley, J. Straits Branch Roy. Asiat. Soc. 75: 31 (1917), p.p.; Saw, Tree Fl. Malaya 4 (1989) 465, p.p.

Small tree to $15 \mathrm{~m}$ tall, to c. $15 \mathrm{~cm}$ diameter. Bark smooth, lenticellate, reddish brown; slash inner bark reddish brown; slash wood cream. Twigs terete, drying greyish brown, densely hairy becoming glabrescent or glabrous in older parts. Leaves: buds densely covered with pale golden brown hairs becoming glabrescent to glabrous as leaves expand except on the main and lateral veins; petioles drying dark brown to black, 0.7-1 cm long, c. $0.8 \mathrm{~mm}$ diam., densely covered with golden brown hairs; lamina thickly coriaceous, $6-10 \times 1.7-3.5 \mathrm{~cm}$, elliptic to obovate, base cuneate, apex acute to cuspidate, margin mostly entire to occasionally denticulate, slightly revolute to flat, upper surface smooth, glabrous, drying dark chocolate brown; lower surface glabrous but hairy on main and lateral veins, drying a lighter tan-brown than the upper surface; midrib sunken above, prominent below; lateral veins 7-9, looping near margin, visible above, prominent below, tertiary veins obscure above, prominent below. Flowers unknown. Fruits ovoid with pointed apex, calyx tube persistent and prominent, sparsely hairy becoming glabrescent, pale green ripening purple, c. $7-8 \times 6-7 \mathrm{~mm}$, smooth and prominently sulcate, pedicel $2-3 \mathrm{~mm}$ long.

Distribution. Endemic to Peninsular Malaysia (Perak and Pahang). So far recorded on the Main Range (Genting Highlands, Fraser's Hill and Cameron Highlands) and Bukit Larut.

Ecology. Lower montane forest, c. 1000-1600 m elevation.

Provisional IUCN conservation assessment. Vulnerable (VU D2). It is a rare plant of the montane forest with an extent of occurrence (EOO) of $3138 \mathrm{~km}^{2}$ and an area of occupancy (AOO) of $16 \mathrm{~km}^{2}$. These mountain forests are in protected areas, but nevertheless qualifies as Vulnerable under criterion D2 as it has an AOO of less than $20 \mathrm{~km}^{2}$ with fewer than 5 locations.

Additional specimens examined. MALAYSIA: Pahang: Bentung, Genting Highlands, 22 May 1987, Saw FRI 35028 (KEP), ibid., FRI 35029 (KEP); Raub, Fraser's Hill, 27 Aug 1971, Loh FRI 19154 (K, KEP, SING). Perak: without specific location, Scortechini 520 (BM, K, SING).

Notes. It is a rare plant which has only been collected three times while fertile. In the Genting Highlands, saplings are rather common (pers. obs.) in the understorey of the lower montane forest. See the discussion under Polyosma fragrans on the clarification of the types and confusion over that species' identity with this species. 


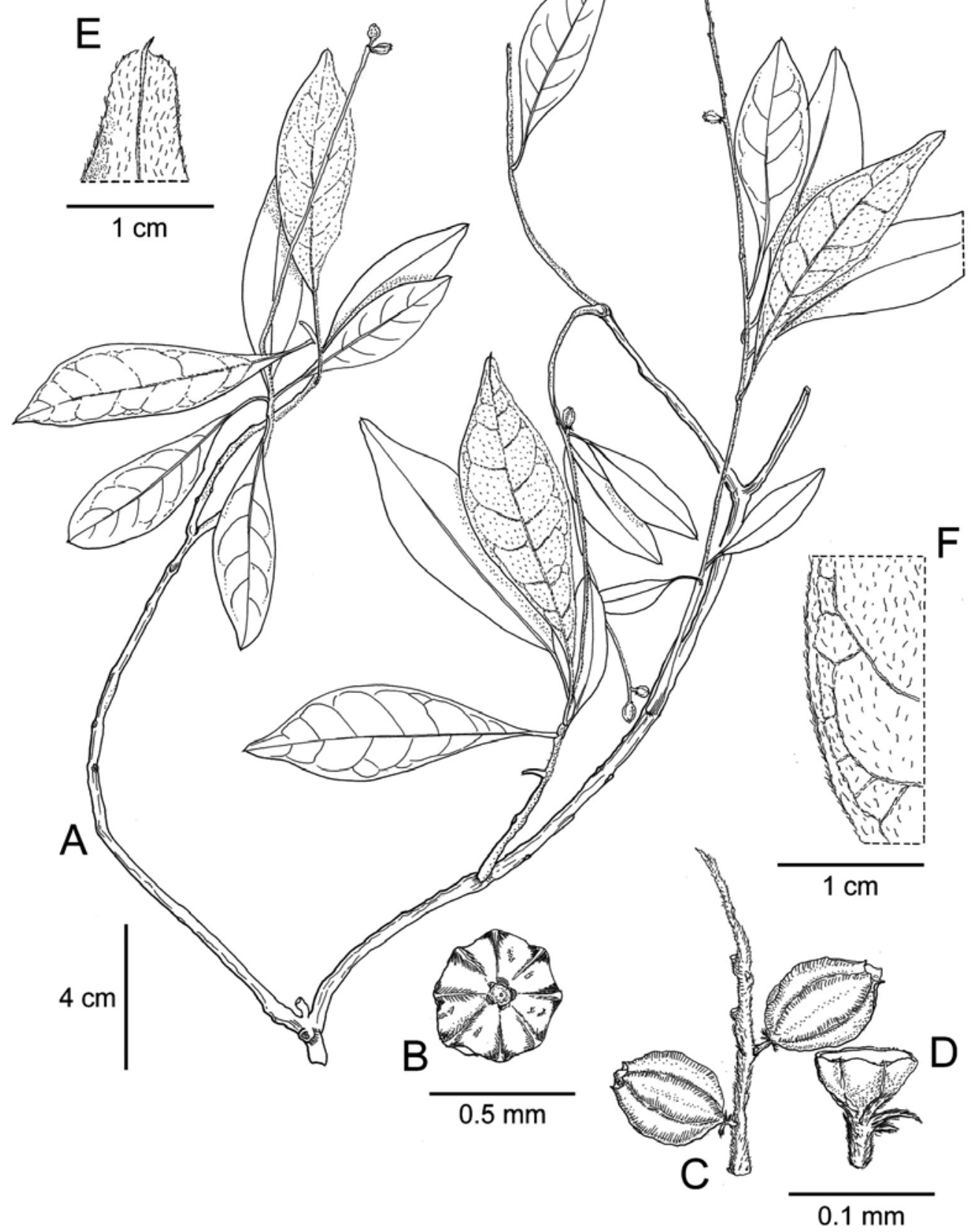

Fig. 1. Polyosma collina Saw. A. Habit. B. Fruit, anterior view. C. Fruits on infructescence stalk. D. Fruit showing bracteoles. E. Leaf tip. F. Leaf margin. A, E \& F from Loh FRI 19154 and B-D from Ogata KEP 110313. Drawn by Mohamad Aidil Nordin. 
2. Polyosma coriacea King, J. Asiat. Soc. Bengal, Pt. 2, Nat. Hist. 66: 300 (1897); Ridley, Fl. Malay Penins. 1: 685 (1922); Saw, Tree Fl. Malaya 4: 465 (1989). - TYPE: Malaysia, Perak, s.d., Scortechini 805 (lectotype K [K000739424], designated here).

Distribution. Endemic in Peninsular Malaysia (recorded from the mountains of Kelantan, Terengganu, Pahang, Selangor and Perak).

Ecology. Montane forest at 1200-1700 m elevation.

Provisional IUCN conservation assessment. Least Concern (LC). The extent of occurrence (EOO) is $35,939 \mathrm{~km}^{2}$, well above the threshold for any threatened categories.

Specimens examined. MALAYSIA: Kelantan: Gn. Rabong, 12 Mar 1972, Soepadmo \& Mahmud 1069 (K, L). Pahang: Bentong, Gn. Mengkuang, 17 Jan 1913, Robinson s.n. (BM, K, L, SING); Cameron Highlands, trail to Gn. Jasar, 2 Mar 1968, Ng FRI 5949 (K, KEP, L); Mentigi FR, Gn. Terbakar, 12 Apr 1934, Symington KEP 36239 (KEP, SING); Sg. Pauh valley, 2 Oct 1963, Chew CWL 740 (K, KEP, L, SAR, SING); Ulu Bertam FR, Gn. Berembun, 27 Oct 1972, Kochummen FRI 16615 (K, KEP, SING), ibid., FRI 16623 (K, KEP, L, SAN, SING); Kuantan, Gn. Tapis, 14 Jun 1934, Symington \& Kiah SFN 28854 (K, KEP); Raub, Fraser's Hill, summit, 22 Apr 1992, Kiew RK 3517 (KEP). Perak: Gn. Inas, May 1892, Wray Jr. 4131 (BM, P); Gn. Kerbau, 9 Mar 1913, Robinson s.n. (K, SING); ibid., 10 Mar 1913, Robinson s.n. (K, SING); ibid., Jun 1913, Robinson s.n. (SING); Kuala Kangsar, Bubu FR, 20 Apr 1995, Chua et al. FRI 39094 (KEP, L); Gn. Bubu, 10 Jun 1978, Mhd Shah \& Mahmud MS 3983 (KEP, SING); ibid., Mar 1890, Wray Jr. 3855 (SING). Terengganu: Besut, Gn. Lawit, 28 Apr 1968, Cockburn FRI 8214 (KEP, L, SING).

3. Polyosma fasciculata Ridl., J. Straits Branch Roy. Asiat. Soc. 75: 31(1917); Ridley, Fl. Malay Penins. 1: 685 (1922); Saw, Tree Fl. Malaya 4: 465 (1989). - TYPE: Malaysia, Kedah, Kedah Peak [Gn. Jerai], June 1893, Ridley 5219 (lectotype SING, designated here; isolectotype BM [BM000611312]).

Distribution. Endemic on Gn. Jerai, Kedah, Peninsular Malaysia.

Ecology. Montane forest over 1000 m elevation.

Provisional IUCN conservation assessment. Vulnerable (VU D2). It is only known from a single location with an area of occupancy (AOO) of only $12 \mathrm{~km}^{2}$. Gn. Jerai is a virgin jungle reserve and is a totally protected area. However, any major threat in this area could drive this species to critically endangered (CR) or extinct (EX) in a very short time, thereby qualifying it for VU D2.

Specimens examined. MALAYSIA: Kedah: Yan, Gn. Jerai, 13 August 1974, Kochummen, FRI 16792 (K, KEP, L, SING); ibid., 30 Mar 1982, FRI 29484 (K, KEP, L, SAN); ibid., 25 Feb 1960, KEP 79129 (KEP); ibid., 8 Nov 1962, Samsuri SA 292 (K, SING), 296 (SING); ibid., 8 May 1938, Symington KEP 46909 (K, KEP). 
4. Polyosma fragrans (Wall.) Benn., P1. Jav. Rar. 196 (1840); Clarke in Hooker, F1. Brit. India 2: 408 (1879); King, J. Asiat. Soc. Bengal, Pt. 2, Nat. Hist. 66: 302 (1897); Ridley, J. Straits Branch Roy. Asiat. Soc. 75: 31 (1917), p.p.; Ridley, Fl. Malay Penins. 1: 684 (1922), p.p. - Itea ? fragrans Wall. in Roxburgh, Flora Ind. 2: 420 (1824). - Polyosma conocarpa Ridl., J. Straits Branch Roy. Asiat. Soc. 75: 31 (1917), nom. illeg., p.p.; Ridley, Fl. Malay Penins. 1: 684 (1922). - TYPE: Singapore, 1822, Wallich s.n. [EIC 8472] (lectotype K-W [K001125569], designated here; isolectotypes B [B 10 0296086], BM [BM000630481], BR [593753], G [G00388959], K [K000739426, K000739427, K000739428, K000739429, K001125568, K001125570], P [P00709653], TCD, W).

Polyosma mutabilis Blume, Mus. Bot. 1: 258 (1850), syn. nov.; King, J. Asiat. Soc. Bengal, Pt. 2, Nat. Hist. 66: 301 (1897); Koorders \& Valeton, Meded. Lands Plantentuin 33: 388 (1900); Beaman et al., Pl. Mount Kinabalu 4: 276 (2001); Gardner et al., Heritage Trees of Penang: 313 (2011); Esser \& Saw, Phytotaxa 221: 89 (2015); Gardner et al., Forest Trees of Southern Thailand 1: 553 (2015), 702; Esser, F1. Thailand 13(3): 540 (2017). - TYPE: Indonesia, Borneo, G. Sakoembang, [1836], Korthals s.n. (lectotype L [L0035107], designated by Esser \& Saw (2015); isolectotypes L [L.1867975, L.1867977]).

Polyosma arguta Craib, Bull. Misc. Inform. Kew 1929: 110 (1929), syn. nov.; Craib, Fl. Siam. 1: 583 (1931). - TYPE: Thailand, Koh Chang, Kao Faimai, 5 June 1925, Rabil 7 (lectotype K, designated by Esser \& Saw (2015); isolectotypes BK [BK257864], BM [BM000600373], TCD [TCD0016603]).

Polyosma bracteolata Craib, Bull. Misc. Inform. Kew 1929: 111 (1929), syn. nov.; Craib, Fl. Siam. 1 (1931) 583. - TYPE: Thailand, Peninsular, Surat Thani, Koh Samui, 9 April 1927, Kerr 12561 (lectotype K, designated by Esser \& Saw (2015); isolectotypes BK [BK214152], BM [BM000600306]).

Polyosma oblonga Craib, Bull. Misc. Inform. Kew 1929: 112 (1929), syn. nov.; Craib, F1. Siam. 1: 585 (1931). - TYPE: Thailand, Nakhon Si Thammarat, Sichon, 13 May 1928, Kerr 15673 (lectotype K, designated by Esser \& Saw (2015); isolectotypes BK [BK257865], BM [BM000600374], TCD [TCD0016598]).

Polyosma sp. 2, Saw, Tree Fl. Malaya 4: 469 (1989).

Distribution. Thailand, Peninsular Malaysia, Singapore, Sumatra, Java, Borneo and Palawan. This is the most widespread Polyosma species globally.

Ecology. Lowland forest to c. 300 m elevation, often near streams or riverbanks.

Provisional IUCN conservation assessment. Least Concern (LC), its extent of occurrence (EOO) is over 4 million $\mathrm{km}^{2}$. 
Specimens examined. MALAYSIA: Johor: Tebrau River, Mar 1907, Ridley s.n. (SING); Mersing, Menchali FR, 25 Feb 1976, Teo 3189 (K, KEP, L, SING). Kedah: Langkawi, Bt Sawar FR, 11 Dec 1969, Whitmore FRI 15017 (K, KEP, L, SAN); Gn. Raya, Feb 1894, Ridley 15524 (BM, K, SING); ibid., Feb 1911, Mhd Haniff 15524 (BM, SING); Gn. Raya FR, 8 Feb 2011, Imin et al. FRI 74601 (E, K, KEP [3 sheets], L, SAN); ibid., 19 Mar 2007, Siti Munirah, et al. FRI 55377 (A, K, KEP, L, SAN, SING); Ma-Chinchang FR, 16 Mar 1969, Chan FRI 6795 (K, KEP, L); Telaga Tujuh, 24 Feb 1983, Kochummen FRI 26089 (K, KEP, L, SAN); Yan, Gn. Jerai, 11 Mar 1971, Kochummen FRI 16324 (K, KEP, L, SAR); Jerai F.R., 4 Apr 1950, Unknown KEP 73514 (KEP); Jerai F.R., Kedah Peak, 26 Feb 1960, Kochummen KEP 94407 (KEP). Kelantan: Chaning, Feb 1917, Ridley 13019 (SING); ibid., Feb 1917, Ridley s.n. (BM, K, SING); Machang, Sg. Jeram Tinggi, Bt. Baka, 24 Feb 1974, Mhd Shah \& Ahmad Shukor MS 3199 (KEP [2 sheets], SAR). Malacca: 1845, Griffith 2510 (K, L); ibid., Griffith s.n. (BM, K, L); ibid., 14 Mar 1867, Maingay 2432 (K). Pahang: Lesong FR, Sg. Jekatih, 20 Feb 1971, Whitmore FRI 15929 (K, KEP); Menchali F.R., 5 May 1982, Khairuddin FRI 31758 (K, KEP, L, SAN); Sg. Bera, 18 Mar 1939, Marshall KEP 35817 (KEP); Kuantan, Tasik Terepai, Chini, 24 May 1919, Forest Ranger Lambok 2725 (K, KEP); Ulu Sg. Riau, 15 Jun 1921, Mahamod KEP 3734 (KEP, SING); Pekan, Sg. Bebar, 11 Apr 2005, Low et al. LYW 42 (L). Penang: Balik Pulau, 1905, Fox 12740 (BM); Pantai Acheh via Telok Bahang, 12 Jan 1983, Stone \& Muhmud 15393 (L); Penang Botanical Garden, 13 Sep 1966, Hou 825 (K, KEP); ibid., 8 Oct 1951, Sinclair SFN 39261 (E, K, L, SING); Penang Hill, Jul 1894, Curtis 1081 (BM, K, SING); Penang Hill, Botanic Gardens, 8 Mar 1991, Kamarudin FRI 34620 (L); Teluk Bahang, Noh s.n. (SING); Timur Laut, Penang Botanic Gardens, 29 Sep 1966, Cheong s.n. (SING); ibid., 2009, Gardner \& Sidisunthorn MY 1 (KEP). Perak: Larut, Jun 1883, Kunstler 4344 (K, L). Terengganu: Sg. Lah, via Bt. Bongkok, 2 Mar 1976, Mhd Shah \& Samsuri MS 3829 (KEP); Besut, Setiu-Kota Baru Road, 28 Jun 1989, Kamarudin FRI 31438 (KEP).

Notes. Polyosma fragrans (Wall.) Benn., originally described as Itea ? fragrans Wall. in Flora Indica (Roxburgh, 1824), was based on Wallich s.n. (East India Company herbarium number 8472 - hereafter referred to as Wallich 8472). Wallich was not certain as to the placement of the species and he provisionally placed it under Itea. Bennett (1840) correctly moved the species to Polyosma. Wallich 8472 contains flowering specimens with several duplicates. However, complications started with the suggestion that Wallich 8472 was a mixed collection (King, 1897; Ridley, 1917). Duplicates of Wallich 8472 include multiple specimens within the same sheets in K (4 sheets) and distributed elsewhere. They contain flowering specimens with leaves of variable sizes. The smaller-leaved specimens were attributed to Polyosma fragrans while the larger-leaved specimens to P. mutabilis Blume. I have examined these and determined that the inflorescences and flowers on these specimens are similar. They all belong to the same species with the name Polyosma fragrans having priority. With this observation, several names are now synonymised here.

I have now placed Polyosma mutabilis into synonymy of $P$. fragrans. The fruits of the type specimen are slightly sulcate and the leaves of thicker texture but they are within the range I have observed for P. fragrans. Esser \& Saw (2015) synonymised three Thai species, P. arguta, P. bracteolata and P. oblonga, into P. mutabilis. With the synonymisation of Polyosma mutabilis into $P$. fragrans, these names are now also subsumed and become new synonyms of $P$. fragrans. 
Ridley (1917) distinguished Polyosma conocarpa Ridl. on fruit characters. He cited several specimens including Wallich 8472 for the new species. As a result, Esser \& Saw (2015) concluded that Polyosma conocarpa Ridl. is illegitimate and superfluous under Art. 52.2 of the ICN (Turland et al., 2018). Ridley (1917) also cited the specimen Wray Jr. 508 under Polyosma conocarpa but this is Polyosma grandis Ridl. With these clarifications, Polyosma fragrans is a lowland species with a wide distribution in Thailand, Peninsular Malaysia, Singapore, Sumatra, Java, Borneo and Palawan.

In the same publication, Ridley (1917) cited two other specimens under Polyosma fragrans (Haviland 944 and Haviland 1886 from Sarawak), which are both P. havilandii Schltr. Polyosma sp. 2 in the Tree Flora of Malaya (Saw, 1989) is part of the variation of $P$. fragrans.

5. Polyosma glaucescens Ridl., J. Straits Branch Roy. Asiat. Soc. 75: 31 (1917); Ridley, Fl. Malay Penins. 1: 684 (1922); Saw, Tree Fl. Malaya 4: 466 (1989). - TYPE: Malaysia, Pahang, Gn. Tahan, 8 July 1905, Wray Jr. \& Robinson 5493 (lectotype K [K000739425], designated here; isolectotype BM [BM000611315]).

Distribution. Endemic in Pahang, Peninsular Malaysia (only known from Gn. Tahan and its neighbouring peaks).

Ecology. Montane forest, 1200-1500 m elevation.

Provisional IUCN conservation assessment. Vulnerable (VU D2). It has a narrow distribution with an extent of occurrence (EOO) of only $9 \mathrm{~km}^{2}$ and an area of occupancy (AOO) of $16 \mathrm{~km}^{2}$. It is distributed in a national park, Taman Negara, which is a totally protected area, but can qualify as Vulnerable due to its restricted AOO with its single-location distribution under criterion D2. A plausible future threat could drive the species to CR or EX in a very short time.

Specimens examined. MALAYSIA: Pahang: Gn. Tahan, Jul 1911, Ridley 16018 (BM, K), Ridley 16019 (BM, K), Ridley 16260 (BM, K); ibid., 24 Feb 1968, Whitmore FRI 4894 (K, KEP, L); ibid., 31 Jun 1905, Wray Jr. \& Robinson 5388 (BM, K); Jerantut, Taman Negara, Gn. Reskit, 10 May 2008, Lim \& Kueh FRI 64850 (KEP, SAN, SING); Lipis, Taman Negara, Kem Belumut, 7 May 2008, Yao \& Kueh FRI 65292 (KEP, SAN).

6. Polyosma grandis Ridl., J. Straits Branch Roy. Asiat. Soc. 75: 30 (1917); Ridley, F1. Malay Penins. 1: 686 (1922). - TYPE: Malaysia, Perak, Larut Hills, January 1883, King 's collector 3802 (lectotype SING [SING0229861], designated here; isolectotypes BM [BM000611316], K [K000739442], P [P00709654], SING [SING0229859, SING0229860]). 
Polyosma integrifolia auct. non Blume: King, J. Asiat. Soc. Bengal, Pt. 2, Nat. Hist. 66: 302 (1897); Saw, Tree Fl. Malaya 4: 466 (1989).

Polyosma conocarpa Ridl., J. Straits Branch Roy. Asiat. Soc. 75: 31 (1917), nom. illeg., p.p. (not including type); Ridley, Fl. Malay Penins. 1: 684 (1922).

Polyosma sp. 3, Saw, Tree Fl. Malaya 4: 469 (1989).

Distribution. Distributed in Peninsular Malaysia and Sumatra, Indonesia. In Peninsular Malaysia recorded from mountain ranges in the states of Pahang (Cameron Highlands, Genting Highlands and Fraser's Hill) and Perak (Larut Hill).

Ecology. Montane forest, 1000-1500 m elevation.

Provisional IUCN conservation assessment. Least Concern (LC). Its extent of occurrence (EOO) is $93,632 \mathrm{~km}^{2}$.

Specimens examined. MALAYSIA: Pahang: Bentung, Genting Highlands, 14 Jun 1967, Whitmore FRI 3883 (K, KEP, L, SAR); Cameron Highlands, 9 Apr 1930, Henderson SFN 23482 (K, KEP); ibid., 2 Apr 1937, Mohd. Nur SFN 32819 (K, KEP [2 sheets],); Gn. Siku FR, 27 Sep 2012, Imin et al. FRI 77541 (BKF, K, KEP, L, SAN, SING); ibid., 20 Feb 2008, Rafidah \& Imin FRI 55645 (KEP); Parit Falls, 25 Jul 1977, Kochummen FRI 18096 (K, KEP, L, SAN, SING); ibid., 6 Aug 2018, Saw et al. JKB 2372 (KEP); Sg. Pauh valley, 4 Oct 1963, Chew CWL 801 (K, KEP, L); Raub, Fraser's Hill, 4 Jun 1972, Stone 10773 (K, L); Fraser's Hill, Girdle Road, Peninjau, 26 Aug 1959, Burkill et al. HMB 2042 (K, L, SAR); Fraser's Hill, Girdle Road, 24 Apr 1992, Kiew RK 3539 (KEP); Fraser's Hill, Methodist's Mission, 26 Aug 1959, Mhd Shah MS 672 (K, L, SAR). Perak: Birch's Hill, Jan 1884, Wray Jr. 508 (K); Larut, Bt. Larut, 12 Mar 1996, van Balgooy 7193 (L); Larut \& Matang, Bt. Larut FR, Maxwell Hill, 31 Dec 2009, Gardner \& Sidisunthorn MY 171 (KEP).

Notes. In the past, this species was confused with Polyosma integrifolia Blume (Clarke, 1879; King, 1897; Saw, 1989) as the leaves are similar in leaf texture and hairiness, and the inflorescence and flower in hairiness. The fruits, however, are different: Polyosma grandis has larger fruits (9-15 $\mathrm{mm}$ long) and these have strongly sulcate endocarps, whilst $P$. integrifolia has smaller fruits (7-9 $\mathrm{mm}$ long), and the endocarps are not sulcate. The leaves are rather variable with some specimens having rather large leaves (e.g. Chew CWL 801, FRI 18096, FRI 55645, FRI 77541, and Gardner \& Sidisunthorn $M Y$ 171) which fall within the variation of the taxon as a whole. In the Tree Flora of Malaya account (Saw, 1989), this larger-leaved variation was treated as Polyosma sp. 3. It is included here as part of the variation of Polyosma grandis as we now have more specimens to understand the range of variation and I have personally collected the species and seen the leaf variation in mature trees. Thus, Polyosma integrifolia does not occur in Peninsular Malaysia but it does occur in Thailand (Esser, 2017).

Among the syntypes Ridley (1917) cited for Polyosma conocarpa was Wray Jr. 508 from Birch's Hill (Larut Hill). This specimen is Polyosma grandis which Ridley himself described from this hill in the same publication. 
7. Polyosma kingiana Schltr., Bot. Jahrb. Syst. 52: 135 (1914); Saw, Tree Fl. Malaya 4: 466 (1989). - TYPE: Malaysia, Perak, Larut, December 1882, King's collector 3685 (lectotype B [B 10 0296085], designated here; isolectotypes BM [BM000906276], G [G00388953], K [K000739431], P [P00709657]).

Polyosma velutina auct. non Blume: King, J. Asiat. Soc. Bengal, Pt. 2, Nat. Hist. 66: 304 (1897).

Polyosma flavovirens Ridl., J. Straits Branch Roy. Asiat. Soc. 75: 32 (1917); Ridley, Fl. Malay Penins. 1: 686 (1922). - TYPE: Malaysia, Penang, Balik Pulau, s.d., Curtis 1165 (lectotype K, designated here; isolectotype BM).

Distribution. Widespread in Peninsular Malaysia (recorded from Kelantan, Kedah, Perak, Pahang, Selangor, Negeri Sembilan), and also found in Singapore.

Ecology. Lowland to hill dipterocarp forest up to $700 \mathrm{~m}$ elevation.

Provisional IUCN conservation assessment. Least Concern (LC). Its extent of occurrence is $72,409 \mathrm{~km}^{2}$.

Specimens examined. MALAYSIA: Kedah: Jeniang Road, 28 Oct 1938, Kiah SFN 36164 (BM, K, KEP). Kelantan: Sg. Lebir, 28 Oct 1938, Henderson SFN 29634 (K, KEP); ibid., 10 Sep 1967, Whitmore FRI 4342 (K, KEP, L); Sg. Perias, 24 Jul 1867, Whitmore FRI 4203 (K, KEP, L). Kuala Lumpur: Weld Hills, 14 Apr 1918, Hamid KEP 1667 (KEP). Negeri Sembilan: Senawang, 31 Feb 1919, Yakim KEP 1993 (K, KEP [2 sheets]). Pahang: Lesong FR (West), near Sg. Jekati, 20 Feb 1971, Samsuri \& Ahmad Shukor SA 529 (KEP); Pekan, Gn. Lesong, Ulu Sepayang FR, Pekan, 12 Oct 1926, Mohamed KEP 8124 (E, KEP). Penang: Feb 1881, Kunstler 1352 (K); Balik Pulau, Mar 1892, Curtis 1165 (BM, K); Government Hill, Feb 1887, Curtis 1165 (K); Penang Hill, Moniot Road East, 18 Oct 1951, Sinclair SFN 39347 (E, K, L). Perak: no location details, 1896, Scortechini 2111 (E); Bruas, Dinding, Feb 1891, Ridley 8930 (K); Larut, Jun 1883, Kunstler 4362 (BM, K); Hulu Perak, Temengor FR, 19 May 2010, Wilkie et al. FRI 72006 (KEP); Sungai Siput, Chior FR, 8 Oct 1967, Ng FRI 5795 (K, KEP, L); ibid., 8 Oct 1967, FRI 5796 (K, KEP, L). Selangor: Bangi FR, 7 Nov 1970, Kochummen FRI 16245 (KEP, L); Sg. Buloh FR, 9 Aug 1937, Symington KEP 44088 (K, KEP, L); Gombak, Bkt. Lagong F.R., 20 May 1947, Wyatt-Smith KEP 52269 (KEP); Bkt. Lagong Sample Plot, Jun 1971, Kochummen 1096 B (KEP); Ginting Simpah, 21st mile, 6 May 1927, Strugnell KEP 12724 (KEP); Hulu Langat, Gn. Nuang, 12 May 1940, Symington KEP 51756 (KEP); Petaling, Sg. Buloh F.R., 26 Mar 1928, Jaamat KEP 15301 (KEP); ibid., 14 Apr 1923, Kiai KEP 8296 (KEP); ibid., 16 Oct 1940, Sow \& Tachun KEP 51849 (KEP); ibid., 16 Oct 1940, KEP 51850 (KEP); Rawang, Kanching FR, 27 Sep 1927, de Sylva KEP 13951 (KEP); Rawang, 30 Jul 1929, Symington KEP 18194 (KEP).

SINGAPORE: Bukit Timah Reserve: 4 Oct 1938, Ngadiman SFN 35910 (BM, E, KEP, L, SING).

Notes. The leaves of Polyosma kingiana can sometimes be confused with P. latifolia from Borneo in their similar size, shape and leaf hairiness. However, their fruits are 
very different: $P$. kingiana has strongly sulcate-turbinate fruits while $P$. latifolia has broadly ellipsoid to globose fruits that are not sulcate.

8. Polyosma laetevirens Griff. ex C.B.Clarke in Hooker, F1. Brit. India 2: 409 (1879); King, J. Asiat. Soc. Bengal, Pt. 2, Nat. Hist. 66: 303 (1897); Ridley, F1. Malay Penins. 1: 685 (1922); Saw, Tree Fl. Malaya 4: 467 (1989); Beaman et al., Pl. Mt. Kinabalu 4: 274 (2001). - TYPE: Malaysia, Malacca, s.d., Griffith 2508 (lectotype K [K000739438], designated here; isolectotypes B [B 10 0296084], GH [00106928], K [K000739437], M [M0242295], P [P00709658]).

Distribution. Peninsular Malaysia (Kelantan, Terengganu, Penang, Perak, Selangor, Melaka and Johor) and Borneo (Sabah, Sarawak and Brunei).

Ecology. Lowland forest to montane forest at c. $1700 \mathrm{~m}$ elevation.

Provisional IUCN conservation assessment. Least Concern (LC). Its extent of occurrence (EOO) is $675,764 \mathrm{~km}^{2}$.

Specimens examined. BRUNEI: Belait: Bt. Teraja, 4 Dec 1991, Coode et al. 6904 (BRUN, K, KEP, L, SAN, SING).

MALAYSIA: Johor: Gn. Blumut, 23 Sep 1970, Suppiah FRI 17814 (K, KEP); 23 Sep 1970, FRI 17838 (KEP); Gn. Ledang, 14 Jul 1969, Whitmore FRI 12310 (K, KEP, SAR). Kelantan: Gn. Stong, 13 Aug 1969, Whitmore FRI 12407 (K, KEP); Kampong Gobek, Kerilla Est., 28 Feb 1959, Mhd Shah \& Kadim MS 462 (K). Malacca: Griffith s.n. (BM, K); 1845, Griffith s.n. (K); Maingay 633 (K); Gn. Ledang (Mt. Ophir), 1845, Griffith 2509 (K, P). Penang: Penang Hill, Aug 1885, Curtis 377 (K); ibid., Mar 1886, Curtis 758 (K); ibid., 8 Aug 1940, Nauen SFN 37670 (K). Perak: Tapah Hills F.R., 3 Apr 1971, Loh FRI 17374 (K, KEP, SAN); Ulu Kerling, Apr 1886, Kunstler 8775 (K); Hulu Perak, Gn. Kenderong, 21 Jan 2010, Teo \& Din KL 5698 (KEP). Selangor: Semangko F.R., Aug 1968, Ando et al. AKK 147 (KEP); Sg. Buloh FR, 25 Feb 1919, Abu KEP 2267 (K, KEP); Rawang, Kanching F.R., 1 Aug 1928, Burmah KEP 15377 (KEP). Terengganu: Gn. Tapis, 9 Sep 1968, Cockburn FRI 10985 (K, KEP).

Notes. This species is the only one in this region that dries with a characteristic obovate grey green leaf and with globose fruits.

9. Polyosma nullii B.C.Stone, Malaysian Forester 43(2): 258 (1980); Saw, Tree F1. Malaya 4: 467 (1989). - TYPE: Malaysia, Pahang, Gn. Ulu Kali, July 1970, Null 342 (holotype KLU).

Distribution. Endemic in Peninsular Malaysia, recorded from Pahang (Gn. Ulu Kali, Gn. Mengkuang and Gn. Benom) and Selangor (Gn. Raja, Hulu Selangor).

Ecology. Upper montane forest at 1200-2000 m elevation. 
Provisional IUCN conservation assessment. Vulnerable (VU D2). It is distributed only on mountain peaks, most of which are protected, but due to its restricted distribution with an extent of occurrence (EOO) of $324 \mathrm{~km}^{2}$ and an area of occupancy (AOO) of 20 $\mathrm{km}^{2}$ it can qualify as vulnerable under criterion D2. It is known only from 4 locations. The population on Gn. Ulu Kali is particularly vulnerable to threat as the Genting Highlands casino is located there and a good portion of the peak has already been developed into hotel and casino facilities.

Specimens examined. MALAYSIA: Pahang: Kluang Terbang, 1910, Barus 10880 (K); Bentong, Gn. Mengkuang, 15 Jan 1913, Robinson s.n. (K); ibid., 19 Jan 1913, Robinson s.n. (BM); Gn. Mengkuang, South, 29 Mar 1959, Wyatt-Smith \& Sow KEP 79156 (KEP, L); Gn. Ulu Kali, 20 Jun 1910, Hashim $34 b$ (KEP); ibid., 11 Jun 1973, Mhd Shah \& Mhd Ali MS 2937 (KEP, SING); ibid., 1910, Murdoch 341 (K); ibid., 31 Mar 1979, Stone 14081 (L); ibid., 5 Aug 1979, Stone 14124 (L); ibid., Stone s.n. 31 Mar 1979 (L); ibid., 3 Sep 1970, Whitmore FRI 15407 (KEP, L); ibid., 11 Jan 1972, FRI 20466 (K, KEP, L, SAR, SING); Bentung, Genting Highlands, Gn. Ulu Kali, 19 Mar 1996, Saw FRI 40232 (K, KEP, L, QRS, SAN, SAR); Temerluh, Krau W.R., Gn. Benom, 19 Mar 1967, Whitmore FRI 3300 (KEP); Termerluh, Gn. Benom, 29 Jul 1925, F.M.S. Mus. Collector s.n. (K); 29 Jul 1925, F.M.S. Mus. Collector s.n. (K); Selangor: Hulu Selangor, Gn. Raja, 28 Jan 1994, Perumal et al. FRI 41675 (KEP).

10. Polyosma parviflora King, J. Asiat. Soc. Bengal, Pt. 2, Nat. Hist. 66: 300 (1897); Saw, Tree Fl. Malaya 4: 468 (1989). - TYPE: Malaysia, Perak, Gn. Inas, May 1892, Wray Jr. 4143 (holotype K [K000739423]).

Polyosma ilicifolia auct. non. Blume: Ridley, Fl. Malay Penins. 1: 683 (1922).

Distribution. Endemic in Peninsular Malaysia (Perak, Selangor, Pahang, Terengganu and Kelantan).

Ecology. Montane forest at 1300-1670 m elevation.

Provisional IUCN conservation assessment. Least Concern (LC). Its extent of occurrence is $27,265 \mathrm{~km}^{2}$.

Specimens examined. MALAYSIA: Kelantan: Gn. Rabong, 14 Mar 1972, Whitmore FRI 20680 (K, KEP, L). Pahang: Sg. Terolak, Ulu Telom, 19 Aug 1931, Jaamat KEP 27588 (K, KEP); Bentong, Gn. Mengkuang, 18 Jan 1913, Robinson s.n. (BM, K); Gn. Ulu Kali, Genting Highlands, 14 Mar 1982, Stone BCS 15089 (KEP); Cameron Highlands, 5 Apr 1988, Khairuddin FRI 32718 (KEP); ibid., 6 Apr 1934, Symington KEP 36060 (K, KEP); Cameron Highlands, Bt. Ruil, 6 Oct 1963, Chew CWL 838 (K, L); Gn. Berembun, 1 Mar 1968, Ng FRI 5925 (K, KEP, L); Sg. Bertam, 11 Apr 1934, Jaamat KEP 36267 (KEP); Ulu Bertam FR, Gn. Berembun, 3 Mar 1994, Perumal et al. FRI 41637 (KEP, L). Perak: Gn. Inas, May 1892, Wray Jr. s.n. (K); Gn. Kerbau, 20 Mar 1913, Robinson s.n. (K). Selangor: Ulu Langat, Menuang Gasing, Kloss s.n. (K); Hulu Langat, Gn. Nuang, 12 May 1940, Symington KEP 51773 (KEP). Terengganu: Gn. Padang S., Jun 1937, Moysey \& Kiah s.n. Jun 1937 (K); Gn. Padang, South, Jun 1937, Moysey \& Kiah SFN 33378 (K, KEP). 
Notes. Polyosma parviflora is rather closely related to P. ilicifolia with their similar looking leaves with denticulate margins. I agree with King's observations (King, 1897) when he first described the species. Even with the much larger number of specimens now available, the characters he used to distinguish it from Polyosma ilicifolia are still applicable. Polyosma parviflora is distinct from $P$. ilicifolia by having shorter inflorescences (2.5-4 cm long), fewer flowers in each inflorescence (6-10 flowers), and rachis, ovary and bracteoles that are glabrescent to glabrous, while $P$. ilicifolia has longer inflorescences (6-12 cm long), more flowers (20-34), and rachis, ovary and bracteoles covered with shaggy golden brown translucent hairs.

11. Polyosma ridleyi King, J. Asiat. Soc. Bengal, Pt. 2, Nat. Hist. 66: 304 (1897); Saw, Tree Fl. Malaya 4: 468 (1989). - TYPE: Singapore, 1892, Ridley 3972 (lectotype BM [BM000600389], designated here; isolectotype SING [SING0044752]).

Polyosma laetevirens Griff. ex King var. robusta Ridl., Fl. Malay Penins. 1: 686 (1922), syn. nov. - Polyosma robusta (Ridl.) Saw, Tree Fl. Malaya 4: 468 (1989). - TYPE: Malaysia, Pahang, Gn. Tahan, July 1911, Ridley 16017 (lectotype K [K000739420], designated here; isolectotypes BM [BM000600390], K [K000739419, K000739421]).

Distribution. Peninsular Malaysia (Perak, Pahang and Johor), Singapore, Sumatra and Borneo (Sabah, Sarawak and Kalimantan). The species is more commonly collected in Peninsular Malaysia than in Borneo.

Ecology. Lowland forest to montane forest at c. $1600 \mathrm{~m}$ elevation, also in heath forest.

Provisional IUCN conservation assessment. Least Concern (LC). Currently the species does not seem to be under threat in Peninsular Malaysia but in Singapore it is presumed nationally extinct. Its extent of occurrence (EOO) is over 1.1 million $\mathrm{km}^{2}$.

Specimens examined. MALAYSIA: Johor: Kota Tinggi, Gn. Panti East, 3 Mar 1968, Cockburn FRI 7785 (K, KEP, L); Mersing, Endau-Rompin Park, South Plateau, 17 Apr 1986, Kiew RK 2196 (KEP); north of Gn. Arong FR, 20 Jan 1954, Wyatt-Smith KEP 54247 (K, KEP, L, SING); Labis FR, 27 Apr 1986, Saw FRI 34338 (K, KEP, SAN); Mersing, 11 Sep 1993, Perromat \& Teo KL 4290 (KEP). Pahang: Gn. Tahan, 3 Jul 1905, Wray Jr. \& Robinson 5462 (K); Merapoh, Taman Negara, Gn. Tahan, 6 Aug 1996, Chua et al. FRI 41549 (KEP). Perak: Larut, Mar 1884, Kunstler 5621 (L).

SINGAPORE: 1880, Cantley s.n. (K, SING); Selatar: 1892, Ridley 3972 (BM).

Notes. This is a distinct species with its smooth ellipsoid fruits and leaf under-surface velvety hairy. The inflorescences of specimens collected from Gn. Tahan, Pahang tend to be shorter with almost sessile fruits. They are however within the variation of the species. As I have here included these variations within the species, Polyosma laetevirens var. robusta ( $P$. robusta) is thereby reduced to synonymy of $P$. ridleyi. 
There are some mistakes in the listing of Ridley 16017, the type specimen for Polyosma laetevirens var. robusta. In SING, that same number, Ridley 16017 [SING0090826] was also used for a collection of Pentaphylax euryoides Gardner \& Champ, another collection also made from Gn. Tahan. The specimen in BM was numbered as 1607 , this was obviously a mistake, G.K. Schulz-Menz had already noted this error in his annotation on the specimen in 1976.

12. Polyosma scortechinii King, J. Asiat. Soc. Bengal, Pt. 2, Nat. Hist. 66: 301 (1897); Ridley, Fl. Malay Penins. 1: 685 (1922); Saw, Tree Fl. Malaya 4: 468 (1989). - TYPE: Malaysia, Perak, s.d., Scortechini 1900, (lectotype K [K000739418], designated here; isolectotypes BM [BM000906243], P [P00709663].

Polyosma sp. 1, Saw, Tree Fl. Malaya 4 (1989) 469.

Distribution. Endemic in Peninsular Malaysia (recorded from the mountains of Terengganu, Pahang, and Perak).

Ecology. Montane forest above c. 800 m elevation.

Provisional IUCN conservation assessment. Least Concern (LC). The extent of occurrence (EOO) is $24,154 \mathrm{~km}^{2}$. The montane ecosystems where the species is distributed are mostly protected.

Specimens examined. MALAYSIA: Pahang: Bentong, Genting Highlands Road, 5 Jan 1972, Kochummen FRI 16470 (K, KEP, L); ibid., 13 Dec 1974, Kochummen FRI 23051 (K, KEP, L); ibid., 22 May 1987, Saw FRI 35031 (KEP); Cameron Highlands, Cameron Highlands, 1 Apr 1937, Mhd Nur SFN 32812 (KEP); Raub, Fraser's Hill, 28 Aug 1971, Loh FRI 19169 (K, KEP, L, SAN); ibid., 28 Aug 1971, Whitmore FRI 20420 (K, KEP, L, SAR); ibid., 11 Dec 1961, Yong KEP 98166 (K, KEP, L, SAN, SAR); Fraser's Hill, Girdle Road, 4 May 2007, Chew et al. FRI 53681 (KEP). Perak: Gn. Inas, 11 Dec 1899, Yapp 458 (K); Larut, 1881, King's Collector (Brown) 2600 (K). Terengganu: Ulu Terengganu, Gn. Lawit, 27 Apr 1974, Ng FRI 22045 (K, KEP, L).

Notes. The specimen Ng FRI 22045 collected from Gn. Lawit, Terengganu has rather large leaves. In the Tree Flora of Malaya account (Saw, 1989), it was listed as Polyosma sp. 1 but it is included here as part of the variation of Polyosma scortechinii.

ACKOWLEDGEMENTS. The author is grateful to the curators of A, B, BM, E, K, KEP, KLU, L, M, P, SAN, SAR, SING and TCD for access to and/or the loan of specimens used in the present study. Access to images of type specimens was also made possible at the JSTOR Global Plants, Naturalis BioPortal and Europeana websites. I am grateful to the Ministry of Water, Land and Natural Resources for providing financial support (SPPIII No. P23085100010021 \& P23085100018003) under the Flora of Peninsular Malaysia project which was the focus of 
this study. I am thankful to my former colleagues in the Forest Research Institute Malaysia, particularly Dr Ruth Kiew, Dr E. Soepadmo and Dr Richard Chung for their support and assistance which I gratefully acknowledge. I am also thankful to Dr Hans-Joachim Esser for sharing some of his notes and discussion on the revision of the genus with me. The critical comments from the two reviewers and Dr David J. Middleton, the editor-in-chief of this journal, have helped improve an earlier version of this paper.

\section{References}

APG IV (2016). An update of the Angiosperm Phylogeny Group classification for the orders and families of flowering plants: APG IV. Bot. J. Linn. Soc. 181(1): 1-20.

Bachman, S., Moat, J., Hill, A.W., de la Torre, J. \& Scott, B. (2011). Supporting Red List threat assessments with GeoCAT: Geospatial conservation assessment tool. ZooKeys 150: $117-126$.

Backer, C.A. \& Bakhuizen van den Brink Jr., R.C. (1964). Escalloniaceae. In: Flora of Java, vol. 1, pp. 506-508. Groningen: P. Noordhoff Ltd.

Bennett, J.J. (1840). Polyosma. In: Plantae Javanicae Rariores, pt. 2, pp. 193-196. London: H. Allen et socios.

Blume, C.L. (1826). Polyosma. In: Bijdragen tot de Flora van Nederlandsch Indie, pt. 13, pp. 658-659. Batavia: Ter Lands Drukerij.

Blume, C.L. (1850). Polyosmaceae. In: Museum Botanicum Lugduno-Batavum Exoticarum, vol. 1, pt. 17, pp. 259-261. Leiden: Brill.

Clarke, C.B. (1879). Saxifragaceae. In: Hooker, J.D. (ed.) Flora of British India, vol. 2, pp. 388-411. London: L. Reeve \& Co.

Craib, W.G. (1928). Contributions to the flora of Siam. Additamentum XXIV. Bull. Misc. Inform. Kew 1928(2): 66-72.

Craib, W.G. (1929). Contributions to the flora of Siam. Additamentum XXVI. Bull. Misc. Inform. Kew 1929(4): 109-119.

Elmer, A.D.E. (1913). Philippine Polyosma. Leafl. Philipp. Bot. 5: 1633-1639.

Elmer, A.D.E. (1939). Saxifragaceae. Leafl. Philipp. Bot. 10: 3790-3792.

Esser, H.-J. (2017). Polyosmaceae. In: Flora of Thailand, vol. 13, pt. 3, pp. 538-545. Bangkok: The Forest Herbarium, Department of National Parks, Wildlife and Plant Conservation.

Esser, H.-J. \& Saw, L.G. (2015). A new species of Polyosma (Escalloniaceae/Polyosmaceae) from Thailand and new synonyms. Phytotaxa 221(1): 89-92.

Heine, H. (1953). Diagnoses novae plantarum in Borneo septentrionali a J. et M.S. Clemens Lectarum. Pars II. Mitt. Bot. Staatssaml. München 1(6): 208-207.

IUCN Standards and Petitions Subcommittee (2017). Guidelines for Using the IUCN Red List Categories and Criteria. Version 13. Prepared by the Standards and Petitions Subcommittee.

King, G. (1897). Saxifragaceae. In: Materials for a Flora of the Malayan Peninsula. J. Asiat. Soc. Bengal 66(2): 297-305.

Lundberg (2016a). Polyosmaceae. In: Kubitzki, K., Kadereit, J.W. \& Bittrich, V. (eds) Families and Genera of Vascular Plants, vol. 14, pp. 291-294. Switzerland: Springer International Publishing.

Lundberg (2016b). Escalloniaceae. In: Kubitzki, K., Kadereit, J.W. \& Bittrich, V. (eds) Families and Genera of Vascular Plants, vol. 14, pp. 185-191. Switzerland: Springer International Publishing. 
Merrill, E.D. (1905). New or noteworthy Philippine Plants, III. Saxifragaceae. Publ. Bur. Sci. Gov. Lab. No. 29: 16-17.

Merrill, E.D. (1912). Saxifragaceae. Philipp. J. Sci., C 7: 271-273.

Merrill, E.D. (1915). Saxifragaceae. Philipp. J. Sci., C 10: 5-7.

Merrill, E.D. (1916). Saxifragaceae. Philipp. J. Sci., C 11: 273-274.

Merrill, E.D. (1919). Saxifragaceae. Philipp. J. Sci. 14: 399-401.

Merrill, E.D. (1921). Saxifragaceae. J. Straits Branch Roy. Asiat. Soc., Special No.: 286-287.

Merrill, E.D. (1923). Polyosma. Enum. Philipp. Fl. Pl. 2: 220-221.

Merrill, E.D. (1928). A collection of plants from Sarawak. Saxifragaceae. Sarawak Mus. J. 3: 523-524.

Ridley, H.N. (1917). New and rare Malayan plants. Series IX. Saxifragaceae. J. Straits Branch Roy. Asiat. Soc. 75: 30-33.

Ridley, H.N. (1922). Saxifragaceae. In: The Flora of the Malay Peninsula, vol. 1, pp. 681-687. Amsterdam: A. Asher \& Co.; and Ashford: L. Reeve \& Co.

Roxburgh, W. (1824). Itea. In: Flora Indica, vol. 2, pp. 418-421. Calcutta: W. Thacker \& Co. and London: Allen \& Co.

Saw, L.G. (1989). Saxifragaceae. In: Ng, F.S.P. (ed.) Tree Flora of Malaya, vol. 4, pp. 462-469. Petaling Jaya: Longman Malaysia Sdn. Berhad.

Saw, L.G. (2004). A new species of Polyosma (Escalloniaceae) from Sabah, Malaysia. Sandakania 14: 57-59.

Saw, L.G. (2010). Vegetation of Peninsular Malaysia. In: Kiew, R., Chung, R.C.K., Saw, L.G., Soepadmo, E. \& Boyce, P.C. (eds) Flora of Peninsular Malaysia, ser. 2, Seed Plants, vol. 1, Malayan Forest Records No. 49, pp. 21-45. Kepong: Forest Research Institute Malaysia.

Schlechter, R. (1914). Die Saxifragaceae Papuasiens. Bot. Jahrb. Syst. 52(1-2): 118-138.

Soltis, D.E. \& Soltis, P.S. (1997). Phylogenetic relationships in Saxifragraceae sensu lato: A comparison of topologies based on $18 \mathrm{~S}$ rDNA and rbcL sequences. Amer. J. Bot. 84: 504-522.

Stapf, O. (1894). On the flora of Mount Kinabalu in North Borneo. Saxifragaceae. Trans. Linn. Soc. London, Bot. 4: 148-149.

Stevens, P.F. (2001 onwards). Angiosperm Phylogeny Website. Version 14, July 2017 [and continuously updated since]. http://www.mobot.org/MOBOT/research/APweb/ Accessed 7 Oct 2020.

Stone, B.C. (1980). Additions to the Malayan Flora VII. Malaysian Forester 43: 244-262.

Thiers, B. (continuously updated). Index Herbariorum: A global directory of public herbaria and associated staff. New York Botanical Garden's Virtual Herbarium. http://sweetgum. nybg.org/science/ih/. Accessed 07 Oct 2020.

Turland, N.J., Wiersema, J.H., Barrie, F.R., Greuter, W., Hawksworth, D.L., Herendeen, P. S., Knapp, S., Kusber, W.-H., Li, D.-Z., Marhold, K. et al. (eds) (2018). International Code of Nomenclature for algae, fungi, and plants (Shenzhen Code) adopted by the Nineteenth International Botanical Congress Shenzhen, China, July 2017. Regnum Vegetabile 159. Glashütten: Koeltz Botanical Books. 
Appendix 1. Index Exsiccatae. The number in parentheses refers to the number of the taxon in the text.

Abu KEP 2267 (8), Ando AKK 147 (8).

Barus 10880 (9), Burkill HMB 2042 (6), Burmah KEP 15377 (8).

Cantley s.n. 1880 (11), Chan FRI 6795 (4), Cheong s.n. 29 Sep 1966 (4), Chew FRI 53681 (12), Chew CWL 740 (2), CWL 801 (6), CWL 838 (10), Chua FRI 39094 (2), FRI 41549 (11), Cockburn FRI 10985 (8), FRI 7785 (11), FRI 8214 (2), Curtis 1081 (4), 1165 (7), 1165 (7), 377 (8), 758 (8).

de Sylva, R.O. KEP 13951 (7).

F.M.S. Mus. Collector s.n. 29 Jul 1925 (9), s.n. 29 Jul 1925 (9), Forest Ranger Lambok 2725 (4), Fox 12740 (4).

Gardner MY 1 (4), MY 171 (6), Griffith 2508 (8), 2509 (8), 2510 (4), s.n. 1845 (8).

Hamid KEP 1667 (7), Hashim, M. 34 b (9), Henderson, M.R. SFN 23482 (6), SFN 29634 (7), Hou, D. $825(4)$.

Imin FRI 74601 (4), FRI 77541 (6).

Jaamat KEP 15301 (7), KEP 27588 (10), KEP 36267 (10).

Kamarudin FRI 31438 (4), FRI 34620 (4), Khairuddin FRI 31758 (4), FRI 32718 (10), Kiah SFN 36164 (7), Kiai KEP 8296 (7), Kiew RK 2196 (11), RK 2196 (11), RK 3517 (2), RK 3539 (6), King's Collector (Brown) 2600 (12), King's Collector (Kunstler) 1352 (7), 3685 (7), 3802 (6), 4344 (4), 4362 (7), 5621 (11), 8775 (8), Kloss s.n. (10), Kochummen $1096 B$ (7), FRI 16245 (7), FRI 16324 (4), FRI 16470 (12), FRI 16615 (2), FRI 16623 (2), FRI 16792 (3), FRI 18096 (6), FRI 23051 (12), FRI 26089 (4), FRI 29484 (3), KEP 79129 (3), KEP 94407 (4).

Lim FRI 64850 (5), Loh FRI 17374 (8), FRI 19154 (1), FRI 19169 (12), Low LYW 42 (4).

Mahamod KEP 3734 (4), Maingay 2432 (4), 633 (8), Marshall KEP 35817 (4), Mohamed KEP 8124 (7), Mohamed Haniff 15524 (4), Mohd. Nur SFN 32812 (12), SFN 32819 (6), Mohd. Shah MS 2937 (9), MS 3199 (4), MS 3829 (4), MS 3983 (2), MS 462 (8), MS 672 (6), Moysey SFN 33378 (10), s.n. Jun 1937 (10), Murdoch 341 (9).

Nauen SFN 37670 (8), Ng FRI 22045 (12), FRI 5795 (7), FRI 5796 (7), FRI 5925 (10), FRI 5949 (2), Ngadiman SFN 35910 (7), Noh s.n. (4), Null 342 (9).

Ogata KEP $110313(1)$

Perromat KL 4290 (11), Perumal FRI 41637 (10), FRI 41675 (9).

Rafidah FRI 55645 (6), Ridley 13019 (4), 15524 (4), 16017 (11), 16018 (5), 16019 (5), 16260 (5), 3972 (11), 5219 (3), 8930 (7), s.n. Feb 1917 (4), s.n. Mar 1907 (4), Robinson 5493 (5), s.n. 10 Mar 1913 (2), s.n. 9 Mar 1913 (2), s.n. 17 Jan 1913 (2), s.n. 20 Mar 1913 (10), s.n. 18 Jan 1913 (10), s.n. 15 Jan 1913 (9), s.n. 19 Jan 1913 (9), s.n. Jun 1913 (2).

Samsuri SA 292 (3), 296 (3), SA 529 (7), Saw FRI 34338 (11), FRI 35028 (1), FRI 35029 (1), FRI 35031 (12), FRI 40232 (9), Scortechini 1900 (12), 2111 (7), 520 (1), 805 (2), Sinclair SFN 39261 (4), SFN 39347 (7), Siti Munirah FRI 55377 (4), Soepadmo 1069 (2), Sow KEP 51849 (7), KEP 51850 (7), JKB 2372 (6), Stone 10773 (6), 14081 (9), 14124 (9), BCS 15089 (10), 15393 (4), s.n. 31 Mar 1979 (9), Strugnell KEP 12724 (7), Suppiah FRI 17814 (8), FRI 17838 (8), Symington KEP 18194 (7), SFN 28854 (2), KEP 36060 (10), KEP 36239 (2), KEP 44088 (7), KEP 46909 (3), KEP 51756 (7), KEP 51773 (10).

Teo KL 3189 (4), KL 5698 (8).

Unknown KEP 73514 (4).

van Balgooy 7193 (6).

Wallich 8472 (4), Whitmore FRI 12310 (8), FRI 12407 (8), FRI 15017 (4), FRI 15407 (9), FRI 15929 (4), FRI 20420 (12), FRI 20466 (9), FRI 20680 (10), FRI 3300 (9), FRI 3883 (6), FRI 4203 (7), FRI 4342 (7), FRI 4894 (5). Wilkie FRI 72006 (7), Wray Jr. 3855 (2), 4131 (2), 4143 (10), 508 (6), 5388 (5), 5462 (11), s.n. May 1892 (10), Wyatt-Smith KEP 52269 (7), KEP 54247 (11), KEP $79156(9)$.

Yakim KEP 1993 (7), Yao FRI 65292 (5), Yapp 458 (12), Yong KEP 98166 (12). 
